\title{
Contradictory Effect of Coronary Collateral Circulation on Regional Myocardial Perfusion That Assessed by Quantitative Myocardial Perfusion Scintigraphy
}

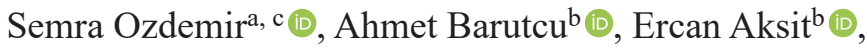 \\ Ali Duygu ${ }^{\mathrm{b}}$ (D), Fulya Koc Ozturk ${ }^{\mathrm{a}}$ (1)
}

\begin{abstract}
Background: Previous studies showed conflicting results about the contribution of coronary collateral circulation (CCC) to myocardial perfusion and function. The aim of this study was to investigate these contradictory problems by gated myocardial perfusion scintigraphy (gated MPS) for the first time.
\end{abstract}

Methods: The current cohort was retrospectively selected among patients who underwent gated MPS and coronary angiography within 2 months. Two different groups including 96 patients were assessed by gated MPS to detect the understanding of the miscellaneous effect of CCC on myocardial perfusion. Group 1 consisted of those who had collateral arteries that were not-well-developed (Rentrop grade $0-1$ ) $(n=58)$, while group 2 consisted of those who had collateral arteries that were well-developed (Rentrop grade 2 - 3) $(n=38)$.

Results: There was no statistically significant difference between groups 1 and 2 in terms of perfusion and functional parameters obtained from gated MPS. Furthermore, no statistically significant difference was found in the phase analysis parameters which is a novel technique to evaluate left ventricular synchronization. On the other hand the left ventricular mass index values were high and quite close to the statistically significant value $(\mathrm{P}=0.059)$ in group 2 .

Conclusions: The current results that obtained by using the gated MPS technique for the first time in the evaluation of CCC showed that the well-developed collateral circulation has a positive effect on myocardial perfusion and function, but this effect was not statistically significant. Results need to be supported by large scale of patients' size.

Manuscript submitted March 28, 2021, accepted April 12, 2021

Published online April 28, 2021

aDepartment of Nuclear Medicine, Faculty of Medicine, Canakkale Onsekiz Mart University, Canakkale 17110, Turkey

bDepartment of Cardiology, Faculty of Medicine, Canakkale Onsekiz Mart University, Canakkale 17110, Turkey

${ }^{\mathrm{c} C}$ Corresponding Author: Semra Ozdemir, Department of Nuclear Medicine, Faculty of Medicine, Canakkale Onsekiz Mart University, Canakkale 17110, Turkey. Email: semozdemir@yahoo.com

doi: https://doi.org/10.14740/cr1262
Keywords: Coronary collateral circulation; Myocardial perfusion scintigraphy; Gated; Phase analysis; Myocardial function

\section{Introduction}

Coronary collateral circulation (CCC) is a natural anastomosis develops as a consequence of severe stenosis or total coronary occlusion in normal coronary circulation [1-3]. The presence of different degrees of collateral circulation in patients with similar levels of coronary artery disease suggests that this important phenomenon is developed by complex mechanisms [4, 5]. Evaluation and grading of CCC depend mainly on invasive procedures such as coronary angiography [6-8]. It is generally thought that the presence of CCC will protect myocardial perfusion and thus prevent ischemia and infarction. Although there are clinical studies showing that the presence of CCC preserves the function of the ventricular and reduce the severity of ischemia and infarction, there are also studies claiming the opposite. Therefore a discussion is still ongoing on this subject $[2,9,10]$. Based on this, we aimed to examine the contribution of CCC to myocardial perfusion and function with gated myocardial perfusion scintigraphy (gated MPS). Gated MPS is a technique in which left ventricular perfusion, motion and synchronization can be evaluated simultaneously. For this reason, the present study was undertaken to examine the effect of coronary collateral presence on left ventricular perfusion, motion and synchronization parameters.

\section{Materials and Methods}

\section{Patient population and protocols}

The study patients were retrospectively selected who underwent myocardial perfusion imaging and coronary angiography within 2 months, between 2018 and 2020. Only the patients with proximal stenosis $\geq 80 \%$ in one or more major coronary artery were included in the study. Retrospective cohort study consisted of 96 patients, 69 of which were men and 27 of which were women (mean age of $63.12 \pm 10.77$ years). Patients with 
Table 1. Demographic and Clinical Parameters of Groups

\begin{tabular}{|c|c|c|c|}
\hline \multirow{2}{*}{ Clinical characteristics } & \multicolumn{2}{|c|}{ Patients cohort $(n=96)$} & \multirow{2}{*}{ P value } \\
\hline & Not-well-developed collateral group $(n=58)$ & Well-developed collateral group $(n=38)$ & \\
\hline The mean age (years) & $64.05 \pm 10.13$ & $61.71 \pm 11.69$ & 0.300 \\
\hline Male & $41(70.7)$ & $28(73.7)$ & 0.750 \\
\hline Female & $17(29.3)$ & $10(26.3)$ & \\
\hline \multicolumn{4}{|l|}{ Comorbidities } \\
\hline Diabetes mellitus, n (\%) & $29(50.0)$ & $11(28.9)$ & $0.041 *$ \\
\hline Hypertension, $\mathrm{n}(\%)$ & $23(39.7)$ & $15(39.5)$ & 0.986 \\
\hline Hyperlipidemia, n (\%) & $33(57.9)$ & $20(52.6)$ & 0.613 \\
\hline Smoking, n (\%) & $28(48.3)$ & $19(50.0)$ & 0.869 \\
\hline Beta blocker & $50(86.2)$ & $31(81.5)$ & 0.516 \\
\hline Vasodilators & $18(31.0)$ & $11(28.9)$ & 0.828 \\
\hline \multicolumn{4}{|l|}{ Coronary angiography } \\
\hline Rentrop 0 (n) & 52 & - & \\
\hline Rentrop 1 (n) & 6 & - & \\
\hline Rentrop 2 (n) & - & 24 & \\
\hline Rentrop 3 (n) & - & 14 & \\
\hline
\end{tabular}

${ }^{*} \mathrm{P}<0.05$. CAD: coronary artery disease.

ejection fraction $(\mathrm{EF}) \leq 40 \%$, organic valvular heart disease, acute decompensated heart failure, coronary artery bypass grafting surgery and permanent pacemakers were excluded from the study. Patients who did not take nitroglycerin during coronary angiography and MPS were included in the study.

The population were divided into two groups; group 1 consisted of those who had collateral arteries that were notwell-developed (Rentrop grade 0 - 1) $(n=58)$, while group 2 consisted of those who had collateral arteries that were welldeveloped (Rentrop grade 2 - 3) $(\mathrm{n}=38)$. Also the patients were divided into three subgroups on the basis of coronary artery types. The MPS studies of all of the selected patients were reprocessed. The clinical characteristics of population and the risk factors for coronary artery disease are given in Table 1.

Written informed consents were obtained from all patients for the MPS imaging. For this study, necessary approvals were received from the local ethics committee (number: 2011-KAEK-27/2019-E.1900102612; date: January 20, 2019). All experiments have been performed in accordance with the ethical standards laid down in the 1964 Declaration of Helsinki.

\section{MPS}

A stress-rest same-day protocol was performed. For the rest studies, 8 to $10 \mathrm{mCi}$ of Tc-99m methoxy-isobutyl-isonitrile (sestamibi) was injected and imaging was performed for 40 min to $1 \mathrm{~h}$. After 3 - $4 \mathrm{~h}$ rest imaging, $24-30 \mathrm{mCi}$ of Tc-99m sestamibi was administered at peak exercise, and imaging was performed for $30 \mathrm{~min}$ to $1 \mathrm{~h}$. Adenosine $(140 \mu \mathrm{g} / \mathrm{kg} / 6 \mathrm{~min})$ or treadmill (Bruce protocol) exercise was used for the myocardial perfusion imaging. Gated MPS was acquired in the supine position using a double-head gamma camera (Infinia, General Electric Medical Systems, MN, USA), which uses $64 \times 64$ matrix and 30 projections. The acquisitions were re-analyzed and semiquantitative analysis was performed using automaticprocessing software for Quantitative Perfusion single-photon emission computerized tomography (SPECT) (QPS), Quantitative Gated SPECT (QGS) and Emory Cardiac Toolbox (ECTb). The SPECT images were analyzed qualitatively and quantitatively by two nuclear medicine specialists.

\section{Quantitative interpretation with QPS and QGS software}

The summed stress score (SSS), summed rest score (SRS) and summed difference score (SDS) were automatically calculated with QPS program. SDS is the difference between the SSS and the SRS, which represents the amount of ischemia $(<2$ : absence of ischemia; 2 - 4: mild ischemia; 5 - 8: moderate ischemia; and $>8$ : severe ischemia).

The summed motion score (SMS) and EF were obtained 
by automated QGS software. The SMS shows myocardial functional movements $(0=$ normal, $1=$ mild hypokinesia, 2 = moderate hypokinesia, $3=$ severe hypokinesia, $4=$ akinesia and $5=$ dyskinesia).

The 17-segment model of the left ventricle (LV) was used for the quantitative scoring of myocardial perfusion and function. The values for all of the segments are summed, giving a total, SSS, SDS and SMS of 68 for the 17-segment model.

\section{Phase analysis with ECTb software}

The transient ischemic dilation (TID) values were calculated using an ECTb program. TID is defined as the apparent presence of left ventricular dilation on post stress images in comparison to the rest images. Patients with a TID ratio (stress/ rest) $>0.98$ are considered to be at high risk in terms of cardiovascular incidents.

The left ventricular mass values were automatically calculated using an ECTb program. The LV mass index (LVMI) was obtained by dividing LVM by body surface area (BSA) (LVM/ BSA, $\mathrm{g} / \mathrm{m}^{2}$ ). BSA was calculated using the following formula: $\mathrm{BSA}=0.6 \times$ height $(\mathrm{m})+0.0128 \times$ weight $(\mathrm{kg})-0.1529$ [11]

Also the phase analysis parameters were automatically calculated using an ECTb program [12, 13]. Three-dimensional count distributions were extracted from each of the LV short-axis images, and were subject to first order Fourier transformation to generation, as three-dimensional phase distribution $\left(0^{\circ}-360^{\circ}\right.$ or $0-100 \%$ RR interval) spanning the entire RR cycle and displayed on a histogram plot. Three quantitative indices were derived from the phase analysis: 1) peak phase (PP), 2) phase standard deviation (PSD), 3) phase histogram bandwidth (PHB). These parameters were used to assess the synchrony of myocardial contraction.

\section{Coronary angiography and coronary collateral grading}

The angiographic images of patients were evaluated retrospectively. The degree of coronary stenosis and the Rentrop grade of collateral circulation were evaluated by two specialist cardiologists, who did not know the clinical characteristics of the patients. A Rentrop scoring system was used to describe collateral circulation, in which $0=$ no collateral vessels, $1=$ poorly opacified collateral vessels without visualization of the epicardial artery, 2 = partial filling of the epicardium by collateral vessels, and $3=$ complete filling of the epicardium by collateral vessels [12]. The collateral circulation was then divided into two groups for data analysis: group 1 was determined as the no or poor collateral circulation group (grade 0 - 1), and group 2 was determined as the good collateral circulation group (grade 2 - 3).

\section{Statistical analysis}

Statistical Package for the Social Sciences (SPSS) version 20.0 was used to perform all statistical analyses, and continuous data were given as mean $\pm \mathrm{SD}$. A paired $t$-test is used to compare two population mean values. After analyzing the normality, Student's $t$-test or Mann-Whitney U test was used to test the differences between the groups in continuous variables. Pearson's Chi-square tests were used to test for the distribution of variables. P values below 0.05 were regarded as statistically significant.

\section{Results}

The study population of the present study consisted of 96 patients, 58 who had not well-developed collateral arteries (group 1), and 38 who had well-developed collateral arteries (group 2). The demographic characteristics of the case groups are summarized in Table 1. Twenty-seven of the patients were female $(28.1 \%)$ while 69 were male $(71.9 \%)$. The median patient age was $61.13 \pm 10.77$ years (range: $34-81$ ). Diabetes was more prevalent in group 1 compared to group $2(50 \% \mathrm{vs}$. $28.9 \%, \mathrm{P}=0.041)$. There were no differences in other clinical characteristics such as age, sex, body mass index, hypertension, hyperlipidemia, smoking and family history of coronary artery disease (Table1).

The variables regarding gated MPS images and coronary angiography are summarized in Tables 2 and 3. The QPS, QGS and $\mathrm{ECTb}$ values obtained from both groups were statistically analyzed to determine the effect of collateral circulation presence on myocardial perfusion and function. In addition, to better understand the real impact of collateral presence, collateralized and non-collateralized subgroups were formed in left artery descending (LAD), circumflex artery (CX) and right coronary artery (RCA). The QPS, QGS and ECTb values of these subgroups were also analyzed in terms of statistical meaning. The results obtained from the study were as follows:

Firstly, there was no significant difference between the two groups in terms of SSS, SDS, values, which are indicators of ischemia and infarction. No significant difference was found in SMS values which are left ventricular function parameters. There were also no differences in the SSS, SDS and SMS values of the coronary artery subgroups. However, even though the data were not statistically significant, it was observed that the SSS, SDS and SMS values of group-subgroups 2 were better than group-subgroups 1 . This results show that CCC had a positive effect on perfusion and movement in the related myocardial area.

Second, stress EF percentage values were examined in both groups and subgroups. The EF values were higher in group 2 and subgroups of 2 . However, this difference was not statistically significant (Tables 2 and 3). This result indicates that the myocardial contraction was partially better preserved in the presence of coronary collaterals.

Third, although TID ratio (stress/rest), which is considered as an indicator of transient ischemia during stress, was lower in group 2 and subgroups of 2, statistical significance was not determined in either of the groups or subgroups (Tables 2 and 3 ).

Fourth, the left ventricular mass values obtained from gamma camera were significantly higher in group 2 compared to group $1(\mathrm{P}=0.011)$. The calculated left ventricular mass 
Table 2. The Statistics of Myocardial Perfusion Scintigraphy Findings in the Current Patients

\begin{tabular}{|c|c|c|c|}
\hline \multirow{2}{*}{$\begin{array}{l}\text { Myocardial perfusion gated } \\
\text { SPECT imaging parameters }\end{array}$} & \multicolumn{2}{|c|}{ Patients cohort $(n=96)$} & \multirow[b]{2}{*}{ P value } \\
\hline & $\begin{array}{l}\text { Not-well-developed col- } \\
\text { lateral group }(\mathrm{n}=58)\end{array}$ & $\begin{array}{l}\text { Well-developed collat- } \\
\text { eral group }(n=38)\end{array}$ & \\
\hline \multicolumn{4}{|l|}{ QPS and QGS } \\
\hline SSS $=$ perfusion damage score & $14.16 \pm 6.41$ & $15.03 \pm 6.96$ & 0.531 \\
\hline $\mathrm{SDS}=$ ischemia score & $7.29 \pm 3.26$ & $7.63 \pm 3.59$ & 0.634 \\
\hline SMS $=$ motion damage score & $11.78 \pm 6.47$ & $11.84 \pm 7.89$ & 0.964 \\
\hline Left ventricular mass (g) & $128.32 \pm 17.65$ & $137.18 \pm 14.36$ & $0.011 *$ \\
\hline Left ventricular mass index $\left(\mathrm{g} / \mathrm{m}^{2}\right)$ & $68.96 \pm 10.29$ & $73.08 \pm 10.40$ & 0.059 \\
\hline TID & $1.11 \pm 0.17$ & $1.06 \pm 0.13$ & 0.158 \\
\hline \multicolumn{4}{|l|}{ Phase analysis (desynchronization scores) } \\
\hline $\mathrm{PP}$ & $147.05 \pm 24.19$ & $138.55 \pm 20.00$ & 0.075 \\
\hline
\end{tabular}

${ }^{*} \mathrm{P}<$ 0.05. SPECT: single-photon emission computerized tomography; QPS: Quantitative Perfusion SPECT; QGS: Quantitative Gated SPECT; SSS: summed stress score; SDS: summed difference score; SMS: summed motion score; EF: ejection fraction; TID: transient ischemic dilation; PP: peak phase; PSD: phase standard deviation; PHB: phase histogram bandwidth.

index values were high in group 2 and quite close to the statistically significant value $(\mathrm{P}=0.059)$.

Lastly, when the phase analysis parameters (PP, PSD, PHB) obtained from the groups were compared, it was determined that even though the values in group 2 were closer to normal values, there were no statistically significant difference in the PP, PSD and PHB values (Table 2). Likewise, although these values were closer to normal in the subgroups of 2 , there was no significant difference between the subgroups regarding the PP, PSD, and PHB values (Table 3).

An example of patient is given in Figure 1. RCA with $100 \%$ total stenosis and Rentrop 3 collateral flow to the RCA from LAD were observed in the angiography technique (Fig. 1a). However in the gated MPS examination the partial positive effect of Rentrop 3 collaterals was observed in the myocardial area of RCA (inferior, inferoseptal walls). Myocardial perfusion bull's eye image of same patient shows infarction + minimal ischemia on the inferior and inferoseptal walls (Fig. 1b).

\section{Discussion}

In this study the relationship between $\mathrm{CCC}$ and left ventricular perfusion, function and synchronization parameters obtained from gated MPS was investigated. No significant statistical difference was found between the groups or subgroups in terms of all MPS parameters representing perfusion and function (SSS, SDS, SMS, EF, TID) and synchronization (phase analysis) indices. Only left ventricular masses were significantly higher in group 2 compared to group 1.

$\mathrm{CCC}$ is defined as potential vessel structures that develop as a chronic response between sections of the same coronary artery or between different coronary arteries when a severe stenosis or complete obstruction occurs in the heart and disrupts blood flow. Many studies have been conducted to understand the mechanism of collateral vessel network development [4, 14]. Previously, it was thought that all coronary collateral vessels already existed and functioned by opening in cases when needed $[6,15,16]$. Today, the development of coronary collateral vessels in the human heart has been shown to be the formation of new vessels (angiogenesis) by budding capillaries from existing blood vessels, as well as arteriogenesis resulting from the enlargement and maturation of the anastomosis channels present between the coronary arteries $[4,17,18]$. The development of CCC is a complex process that is influenced by many factors, and the debate on the formation mechanisms and effects is still ongoing [19-22]. In addition, therapeutic angiogenesis studies aimed at increasing the development of CCC have become a popular research area. Studies with cytokine, stem cell and gene therapies that are effective on CCC development are still ongoing and showing promising results on animal models [5, 23, 24].

On the other hand, it is also important to understand the effects of CCC presence on left ventricular perfusion and function. While there are studies reporting that CCC preserves myocardial perfusion and function and contributes to survival, there are also studies reporting that it does not have as important contribution as expected [2, 25, 26]. For example, Ajayi et al studied patients with total occlusion of a coronary artery and reported that left ventricular function and EF value were significantly higher in patients who had developed well-functioning coronary collaterals [27]. In a study investigating the effects of CCC on left ventricular perfusion and function, it was stated that well-developed coronary collaterals reduced left ventricular dysfunction, infarct size, and mortality [16]. In their study Malek et al determined that myocardial segments supplied by chronic total occlusion with good collaterals were less inclined to inducible ischemia and were less likely to un- 


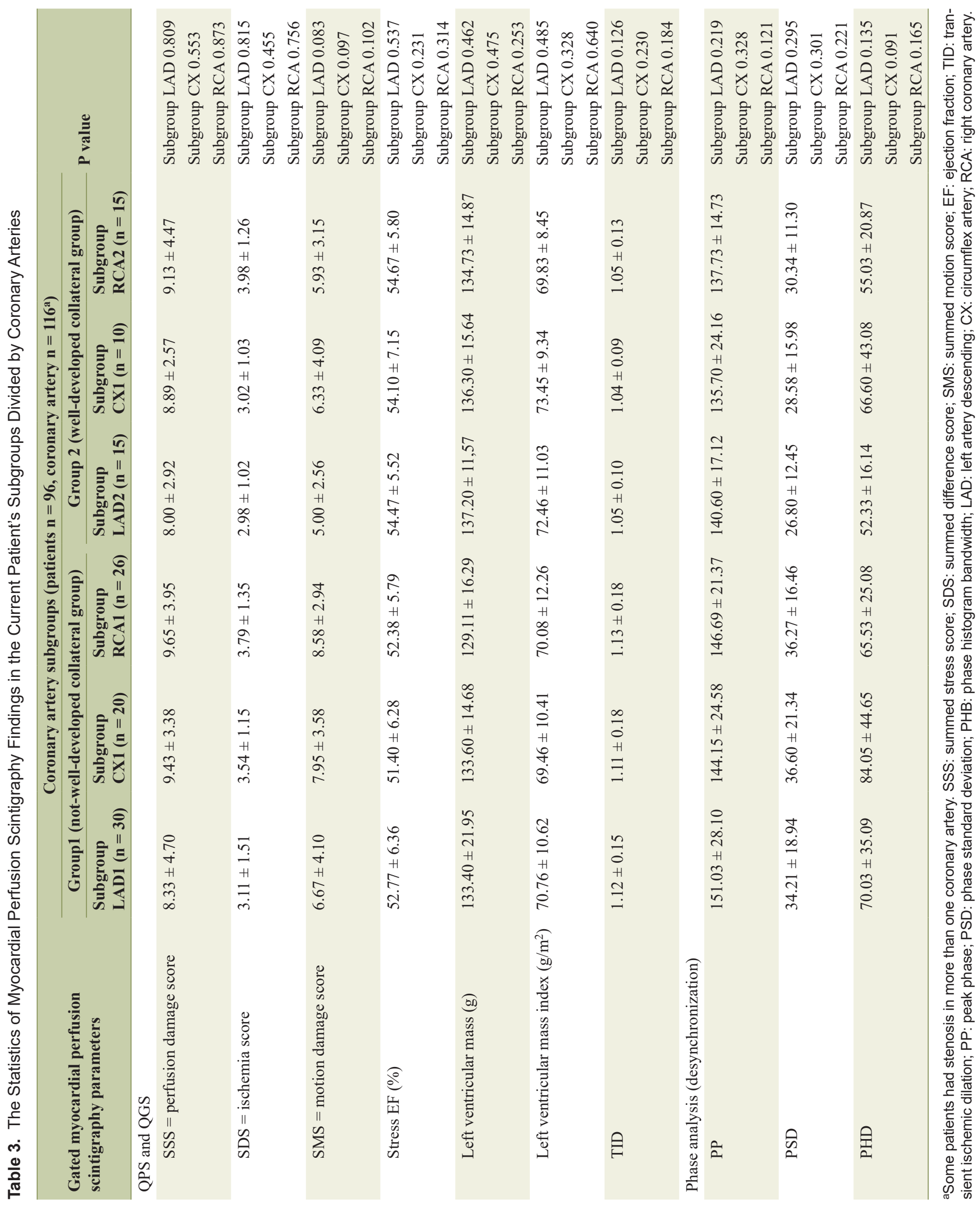




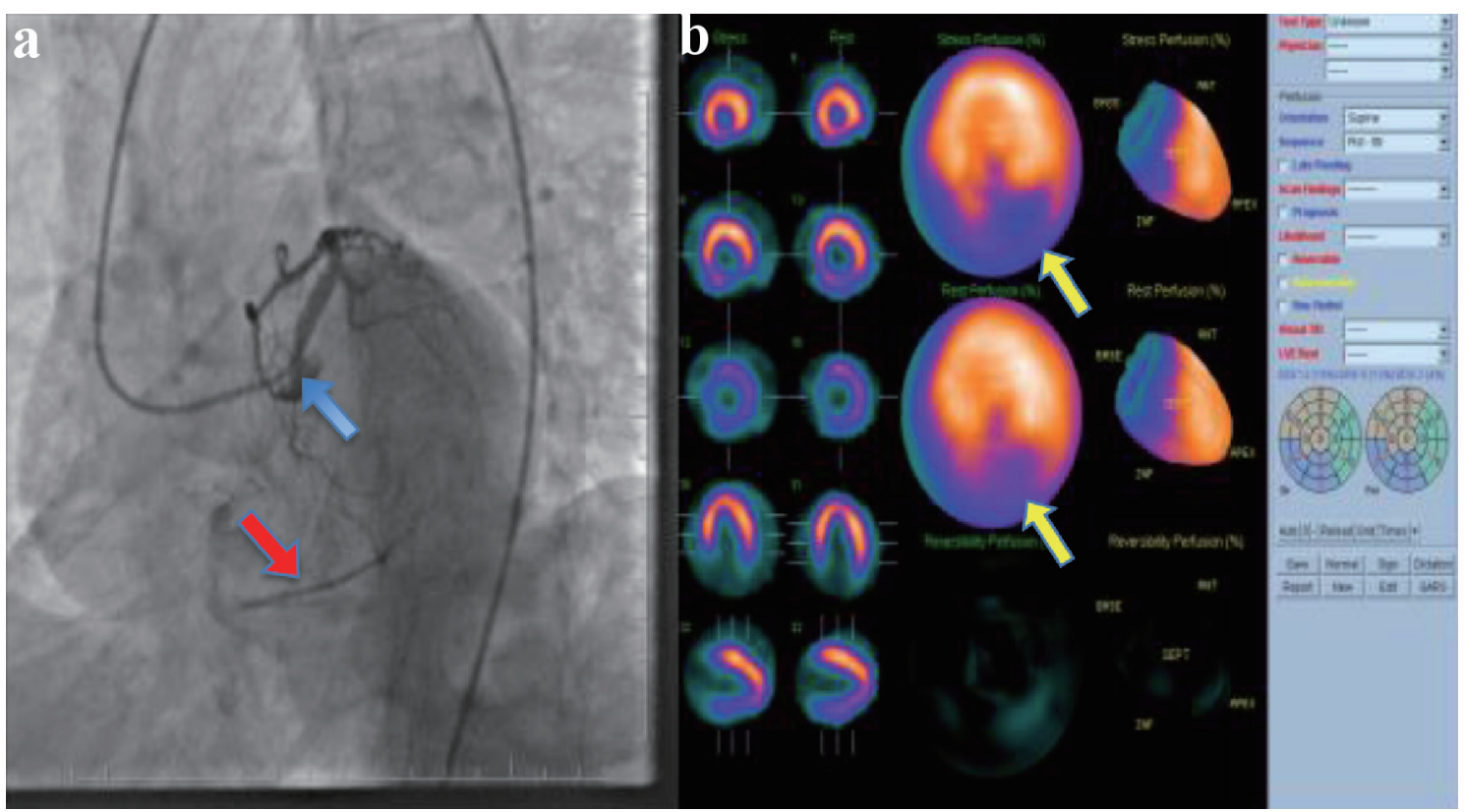

Figure 1. An example of patient. (a) Proximal right RCA with $100 \%$ total stenosis (blue arrow) and Rentrop 3 collateral flow to the RCA from LAD (red arrow). (b) Myocardial perfusion bull's eye image of the same patient shows infarction and minimal ischemia on the inferior wall (yellow arrows). LAD: left artery descending; RCA: right coronary artery.

dergo myocardial infarction [28].

However, there are also studies stating that good collateral development does not contribute to myocardial perfusion and viability. Aboul-Enein et al conducted a study on groups of patients with well-developed collateral and not-well-developed collateral, and reported that there was no significant difference between post-stress myocardial perfusions in either group in MPS [29]. Moreover, in a study in which the effect of collateral presence on myocardial viability in patients with chronic total occluded coronary arteries was investigated with $18 \mathrm{~F}$ fluorodeoxyglucose positron emission tomography/computed tomography (FDG PET/CT), it was reported that CCC had no contribution to myocardial viability [30].

In another study myocardial blood flow and coronary flow reserve measurements were performed with PET in patients with high EF levels and good CCC, and the results suggested that collateral function during increased blood flow requirement in viable myocardium was mostly unsatisfactory [31]. In addition, Hasanovic et al confirmed these results in their study as they reported that the effective angiographic collaterals may prevent resting regional wall motion abnormalities, but do not appear to protect against stress-induced perfusion defect [32]. Similar to the results of this perfusion and viability study, in the present study, perfusion and function parameters were partially preserved in group 2 although they were not statistically significant. In a study conducted by Cetin et al it was suggested that left ventricular hypertrophy increases CCC development [33]. According to the results of the present study, left ventricular mass values were significantly higher in group 2 compared to group 1, and left ventricular mass index values were found to be high and very close to the statistical significance. Although this significant result was not obtained in the subgroups, it was thought that this might be due to the insufficient number of patients in the subgroups.

As previously reported in many studies, the results of the present study also show that the presence of diabetes mellitus (DM) is a negative factor for coronary collateral development $[34,35]$.

The present study also compared left ventricular dyssynchrony parameters obtained from myocardial perfusion imaging between groups. Even though three quantitative parameters, namely PP, PSD, PHB, were reproduced from the phase analysis, only the PSD and PHB parameters could be used to determine mechanical dyssynchrony. Hence, high phase PSD and PHB values remark higher degrees of mechanical dyssynchrony [36]. The present study indicated that the PSD and PHB values were higher in group 1 than in group 2; however the differences were not statistically significant (Tables 2 and 3 ). This result confirms that the presence of CCC, although not statistically significant, reduces the level of dyssynchrony.

\section{Limitations of the study}

The major limitation of this study was the small population size. This study needs to be confirmed by a larger scale of studies to be conducted for the advanced clarifying of effect of $\mathrm{CCC}$ on myocardial perfusion and function. It was thought that statistically significant results could be obtained if the study could be expanded with larger patient series.

\section{Conclusions}

Our study results showed that the well-developed collateral 
circulation has a positive but statistically insignificant effect on myocardial perfusion, function and synchronization. Results need to be supported by large scale of patients' size.

\section{Acknowledgments}

The authors thank the family members who agreed to participate in the presented results.

\section{Financial Disclosure}

No funding was obtained for this study.

\section{Conflict of Interest}

The authors declare that they have no competing interest.

\section{Informed Consent}

Informed written consents were obtained from all patients who participated in this study.

\section{Author Contributions}

Concept and design: SO, AB and EA. Supervision: SO. Material: $\mathrm{SO}, \mathrm{AD}$, and $\mathrm{FKO}$. Data collection and/or processing: $\mathrm{SO}, \mathrm{AD}$, and FKO. Analysis and/or interpretation: $\mathrm{SO}$ and $\mathrm{AB}$. Literature search: SO and EA. Writing manuscript: SO. All authors read and approved the final manuscript.

\section{Data Availability}

All data generated or analyzed during this study are included in this published article.

\section{References}

1. Bigler MR, Seiler C. The human coronary collateral circulation, its extracardiac anastomoses and their therapeutic promotion. Int J Mol Sci. 2019;20(15):3726.

2. Jamaiyar A, Juguilon C, Dong F, Cumpston D, Enrick M, Chilian WM, Yin L. Cardioprotection during ischemia by coronary collateral growth. Am J Physiol Heart Circ Physiol. 2019;316(1):H1-H9.

3. Xing Z, Pei J, Tang L, Hu X. Traditional cardiovascular risk factors and coronary collateral circulation: Protocol for a systematic review and meta-analysis of case-control studies. Medicine (Baltimore). 2018;97(17):e0417.

4. Zimarino M, D'Andreamatteo M, Waksman R, Epstein SE, De Caterina R. The dynamics of the coronary collateral circulation. Nat Rev Cardiol. 2014;11(4):191-197.
5. Potz BA, Parulkar AB, Abid RM, Sodha NR, Sellke FW. Novel molecular targets for coronary angiogenesis and ischemic heart disease. Coron Artery Dis. 2017;28(7):605613.

6. Meier P, Schirmer SH, Lansky AJ, Timmis A, Pitt B, Seiler $\mathrm{C}$. The collateral circulation of the heart. BMC Med. 2013;11:143.

7. Pries AR, Badimon L, Bugiardini R, Camici PG, Dorobantu M, Duncker DJ, Escaned J, et al. Coronary vascular regulation, remodelling, and collateralization: mechanisms and clinical implications on behalf of the working group on coronary pathophysiology and microcirculation. Eur Heart J. 2015;36(45):3134-3146.

8. de Marchi SF. Determinants of human coronary collaterals. Curr Cardiol Rev. 2014;10(1):24-28.

9. Shah A. Chronic Total Occlusion Coronary Intervention: In Search of a Definitive Benefit. Methodist Debakey Cardiovasc J. 2018;14(1):50-59.

10. Choo GH. Collateral Circulation in Chronic Total Occlusions - an interventional perspective. Curr Cardiol Rev. 2015;11(4):277-284.

11. Cuspidi C, Facchetti R, Bombelli M, Sala C, Grassi G, Mancia G. Differential value of left ventricular mass index and wall thickness in predicting cardiovascular prognosis: data from the PAMELA population. Am J Hypertens. 2014;27(8):1079-1086.

12. Rentrop KP, Cohen M, Blanke H, Phillips RA. Changes in collateral channel filling immediately after controlled coronary artery occlusion by an angioplasty balloon in human subjects. J Am Coll Cardiol. 1985;5(3):587-592.

13. Vo MN, Brilakis ES, Kass M, Ravandi A. Physiologic significance of coronary collaterals in chronic total occlusions. Can J Physiol Pharmacol. 2015;93(10):867-871.

14. Jennings RB, Wagner GS. Roles of collateral arterial flow and ischemic preconditioning in protection of acutely ischemic myocardium. J Electrocardiol. 2014;47(4):491499.

15. Werner GS. The role of coronary collaterals in chronic total occlusions. Curr Cardiol Rev. 2014;10(1):57-64.

16. Seiler C, Meier P. Historical aspects and relevance of the human coronary collateral circulation. Curr Cardiol Rev. 2014;10(1):2-16.

17. Fei Y, Hou J, Xuan W, Zhang C, Meng X. The relationship of plasma miR-503 and coronary collateral circulation in patients with coronary artery disease. Life Sci. 2018;207:145-151.

18. Gurses KM, Yalcin MU, Kocyigit D, Besler MS, Canpinar H, Evranos B, Yorgun $\mathrm{H}$, et al. The association between serum angiogenin and osteopontin levels and coronary collateral circulation in patients with chronic total occlusion. Anatol J Cardiol. 2019;22(2):77-84.

19. Stoller M, Seiler C. Salient features of the coronary collateral circulation and its clinical relevance. Swiss Med Wkly. 2015;145:w14154.

20. Liu Z, Pericak-Vance MA, Goldschmidt-Clermont P, Seo D, Wang L, Rundek T, Beecham GW. Coronary collateralization shows sex and racial-ethnic differences in obstructive artery disease patients. PLoS One. 2017;12(10): e0183836. 
21. Kiziltunc E, Gok M, Topcuoglu C, Kundi H, Cetin M, Turhan T, Ornek E. Serum sphingosine 1 phosphate levels in patients with and without coronary collateral circulation. Acta Cardiol Sin. 2018;34(5):379-385.

22. Shen Y, Chen S, Dai Y, Wang XQ, Zhang RY, Yang ZK, $\mathrm{Hu}$ J, et al. Lipoprotein (a) interactions with cholesterolcontaining lipids on angiographic coronary collateralization in type 2 diabetic patients with chronic total occlusion. Cardiovasc Diabetol. 2019;18(1):82.

23. Kaminsky SM, Quach L, Chen S, Pierre-Destine L, Van de Graaf B, Monette S, Rosenberg JB, et al. Safety of direct cardiac administration of AdVEGF-All6A+, a replication-deficient adenovirus vector $\mathrm{cDNA} /$ genomic hybrid expressing all three major isoforms of human vascular endothelial growth factor, to the ischemic myocardium of rats. Hum Gene Ther Clin Dev. 2013;24(1):38-46.

24. Wang Y, Zhang L, Jia L, Liu J, Liu K, Feng Q, Wang Q. Calcitonin gene-related peptide in aerobic exercise induces collateral circulation development in rat ischemia myocardium. Biomed Pharmacother. 2016;82:561-567.

25. Wang L, Lu MJ, Feng L, Wang J, Fang W, He ZX, Dou $\mathrm{KF}$, et al. Relationship of myocardial hibernation, scar, and angiographic collateral flow in ischemic cardiomyopathy with coronary chronic total occlusion. J Nucl Cardiol. 2019;26(5):1720-1730.

26. Meier P, Lansky AJ, Fahy M, Xu K, White HD, Bertrand ME, Mehran R, et al. The impact of the coronary collateral circulation on outcomes in patients with acute coronary syndromes: results from the ACUITY trial. Heart. 2014;100(8):647-651.

27. Ajayi NO, Vanker EA, Satyapal KS. The role of coronary artery collaterals in the preservation of left ventricular function: a study to address a long-standing controversy. Cardiovasc J Afr. 2017;28(2):81-85.

28. Malek LA, Spiewak M, Klopotowski M, Marczak M, Witkowski A. Combined analysis of myocardial function, viability, and stress perfusion in patients with chronic total occlusion in relation to collateral flow. Kardiol Pol.
2015;73(10):909-915.

29. Aboul-Enein F, Kar S, Hayes SW, Sciammarella M, Abi$\operatorname{dov}$ A, Makkar R, Friedman JD, et al. Influence of angiographic collateral circulation on myocardial perfusion in patients with chronic total occlusion of a single coronary artery and no prior myocardial infarction. J Nucl Med. 2004;45(6):950-955.

30. Dong W, Li J, Mi H, Song X, Jiao J, Li Q. Relationship between collateral circulation and myocardial viability of (18)F-FDG PET/CT subtended by chronic total occluded coronary arteries. Ann Nucl Med. 2018;32(3):197-205.

31. Stuijfzand WJ, Driessen RS, Raijmakers PG, Rijnierse MT, Maeremans J, Hollander MR, Lammertsma AA, et al. Prevalence of ischaemia in patients with a chronic total occlusion and preserved left ventricular ejection fraction. Eur Heart J Cardiovasc Imaging. 2017;18(9):10251033.

32. Hasanovic A. Collateral function in patients with coronary occlusion evaluated by 201 thallium scintigraphy. Bosn J Basic Med Sci. 2008;8(4):304-308.

33. Cetin MS, Ozcan Cetin EH, Ocak K, Ozcan Celebi O, Aras D, Temizhan A, Aydogdu S. The association between left ventricular mass index and coronary collateral circulation in patients with chronic total occlusion. Turk Kardiyol Dern Ars. 2019;47(1):21-28.

34. Yetkin E, Topal E, Erguzel N, Senen K, Heper G, Waltenberger J. Diabetes mellitus and female gender are the strongest predictors of poor collateral vessel development in patients with severe coronary artery stenosis. Angiogenesis. 2015;18(2):201-207.

35. Kurtul A, Ozturk S. Prognostic value of coronary collaterals in patients with acute coronary syndromes. Coron Artery Dis. 2017;28(5):406-412.

36. Henneman MM, Chen J, Dibbets-Schneider P, Stokkel MP, Bleeker GB, Ypenburg C, van der Wall EE, et al. Can LV dyssynchrony as assessed with phase analysis on gated myocardial perfusion SPECT predict response to CRT? J Nucl Med. 2007;48(7):1104-1111. 